\title{
PAX8: A Highly Sensitive Marker for the Glands in Extragenital Endometriosis
}

\author{
Tomoko Arakawa, MD', Shinya Fukuda, MD, PhD', Tetsuya Hirata, MD, PhD'®, \\ Kazuaki Neriishi, MD', Yu Wang, MD, PhD', Arisa Takeuchi, MD', \\ Ai Saeki, MD'2, Miyuki Harada, MD, PhD', Yasushi Hirota, MD, PhD', \\ Takashi Matsumoto, MD'2, Kaori Koga, MD, PhD', Osamu Wada-Hiraike, MD, PhD', \\ Masatoshi Kurihara, MD, PhD' ${ }^{3}$, Tomoyuki Fujii, MD, PhD', \\ and Yutaka Osuga, MD, PhD'
}

\begin{abstract}
In cases of extragenital endometriosis or microscopic endometriosis lesions, pathological diagnosis can be challenging because endometriotic stroma and glands represent only a minor component of fibrotic endometriotic lesions. For better accuracy of diagnosis, the development of a sensitive and specific epithelial marker is beneficial. Previous studies showed that PAX8 is a highly sensitive and specific marker for primary and metastatic Mullerian epithelial tumors. Therefore, we sought to examine whether PAX8 is a highly sensitive marker for glands in extragenital endometriosis. Eight and 47 samples of ovarian endometrioma and extragenital endometriosis, respectively, were evaluated in this study. We calculated the percentage of samples positively immunostained for PAX8, CDI0, estrogen receptor (ER), and progesterone receptor (PR). PAX8 was positive for endometriotic epithelial cells in $95.7 \%$ (45/47) of extragenital endometrioses and in 100\% (8/8) of ovarian endometrioses. CDI0 was positive for endometriotic stromal cells in $97.9 \%$ (46/47) of extragenital endometrioses. PAX8 was strongly positive for glands, even in a CD I0-negative case. The expression of PAX8, CD I0, and PR was not affected by preoperative hormonal therapy, and the positive rate of ER staining was significantly reduced by preoperative hormonal therapy. In conclusion, PAX8 is a highly sensitive epithelial marker for extragenital endometriosis. This specific expression was maintained under hormonal therapy. It is noteworthy that extragenital endometriosis maintains the expression of this lineage marker, although it occurs at various sites, and its cause and mechanism of development might be different. PAX8 nuclear expression can be useful in detecting extragenital endometriosis in clinical practice.
\end{abstract}

\section{Keywords}

extragenital endometriosis, marker, PAX8, CDI0

\section{Introduction}

Endometriosis is a common benign gynecological disease affecting approximately $10 \%$ of reproductive-aged women. ${ }^{1}$ Endometriosis commonly forms lesions, such as peritoneal, ovarian, and deep filtrating endometriosis, and less commonly involves the bowel, bladder, or rarely distant sites from the uterus, such as the lung. Pathologic diagnosis of endometriosis is performed with the presence of endometrial-like tissues composed of glandular epithelium and endometriotic stroma, as well as extragenital endometriosis. When it is not clearly diagnosed, it is possible to show the presence of endometriotic stroma by confirming the expression of CD10 by immunostaining, ${ }^{2,3}$ and the expression of estrogen receptor $\alpha$ (ER) and progesterone receptor (PR) is also helpful to the diagnosis of endometriosis. In particular, CD10 was reported to be very sensitive to endometrial and endometriotic stromal cells and is a diagnostically useful immunohistochemical marker of endometriosis. ${ }^{2,3}$ However, in cases of extragenital endometriosis or microscopic endometriosis lesions, pathological diagnosis can be challenging because endometriotic stroma and glands represent only a minor component of fibrotic endometriotic lesions. ${ }^{4}$ Therefore, for better accuracy of diagnosis, the

\footnotetext{
' Department of Obstetrics and Gynecology, University of Tokyo, Tokyo, Japan

${ }^{2}$ Department of Gynecology, Osaka Central Hospital, Tokyo, Japan

${ }^{3}$ Pneumothorax Research Center and Division of Thoracic Surgery, Nissan Tamagawa Hospital, Tokyo, Japan
}

\section{Corresponding Author:}

Tetsuya Hirata, Department of Obstetrics and Gynecology, Faculty of Medicine, University of Tokyo, 7-3-I Hongo, Bunkyo-ku, Tokyo I I3-8655, Japan.

Email: thira-tky@umin.ac.jp 
development of a useful epithelial marker is beneficial. Furthermore, a very recent paper has demonstrated that endometriotic epithelium is clonally and developmentally distinct from stroma in ovarian, intestinal, and uterosacral endometrioses, implying the importance of endometriotic epithelial cells. $^{5}$

PAX8 belongs to the pair-box (PAX) gene family consisting of 9 members (PAX1 to PAX9), each of which encodes a development transcription factor that are key regulators for proper tissue formation and cellular differentiation and are also crucial for maintaining the normal function of cells after birth. ${ }^{6,7}$ PAX8 is essential for embryonic development of the kidney, Mullerian organs, and thyroid. ${ }^{8-10}$ Previous studies showed that PAX8 is a sensitive and specific marker for primary and metastatic Mullerian epithelial tumors, including ovarian and endometrial cancers by comprehensive immunohistochemical analysis. ${ }^{11-13}$ Furthermore, endometrial epithelia and ovarian endometriotic epithelia were also reported to be positive for PAX8. ${ }^{12,13}$ Moreover, the deficiency of the PAX8 gene in mice results in a nonfunctional uterus with the absence of the endometrium. ${ }^{10}$ Given that PAX8 was reported to identify ovarian and Mullerian-derived tumors even in metastatic lesions, ${ }^{12-14}$ we considered that PAX8 might be useful as an epithelial marker of extragenital endometriosis. However, there has been no report on extragenital endometriosis. Here, we sought to comprehensively analyze PAX8 expression in various extragenital endometrioses.

\section{Materials and Methods}

\section{Patients and Samples}

The experimental procedures were approved by the institutional review board of the University of Tokyo (registration no. \#11442). All patients who underwent surgical removal of ovarian endometrioma as well as bowel, bladder, and thoracic endometrioses and diagnosed at the University of Tokyo Hospital, Nissan Tamagawa Hospital, and Osaka Chuo Hospital were enrolled in this study. We obtained written informed consent from the patient who came to the outpatient for postoperative follow-up and whose tissue section was used for this study. The diagnosis of endometriosis and extragenital endometriosis was performed through a histological examination performed by certified pathologists. Available hematoxylin and eosinstained slides were reviewed, and the diagnosis was confirmed in each case. We enrolled 55 patients after reviewing the medical records. We recorded the patient age at the time of the operation, history of pregnancy, parturition, and preoperative hormonal therapy.

Paraffin-embedded specimens were sliced with $5-\mu \mathrm{m}$ thickness, and the sections placed on slides were then deparaffinized and rehydrated. Antigens were retrieved using an antigen retrieval reagent (Immunosaver; Nissin EM, Tokyo, Japan) at $98^{\circ} \mathrm{C}$. Endogenous peroxidase was blocked by incubation for 10 minutes with a solution of $0.3 \%$ hydrogen peroxidase. Immunohistochemical tissue labeling for PAX8 (1:1000,
10336-1-AP; Proteintech, Illinois), CD10 (1:1, IR468; Readyto-use, Agilent, California), ER (1:1, IR084; Ready-to-use, Agilent), and PR (1:1000, \#8757; Cell Signaling Technology, Tokyo, Japan) was performed using the Dako Envision FLEX+ system (Agilent, Santa Clara, California). After blocking with a nonspecific staining blocking reagent (Agilent), the sections were incubated with each specific antibody or rabbit $\operatorname{IgG}$ for 60 minutes at room temperature and then incubated with peroxidase-conjugated secondary antibody for $30 \mathrm{~min}$ utes. Staining was detected using diaminobenzidine chromogen for a few minutes. All sections were counterstained with hematoxylin and evaluated under a light microscope.

\section{Statistical Analysis}

Statistical analysis was performed using JMP Pro version 14 (SAS Institute Inc, Cary, North Carolina). Continuous data were reported as mean and standard deviation. Categorical data were analyzed using $\chi^{2}$ tests, and Fisher exact tests and were presented as counts and percentages. Results were considered statistically significant at $P$ value $<.05$.

\section{Results}

\section{Clinical Characteristics}

Table 1 shows the clinical characteristics of patients with ovarian endometrioma or extragenital endometriosis. Eight ovarian endometriomas and 47 extragenital endometrioses were enrolled in this study, which included 20 intestinal, 10 bladder, 3 ureteral, 3 inguinal, and 11 diaphragmatic endometrioses. The mean age at surgery of patients with ovarian endometrioma and extragenital endometriosis was $36.0 \pm 4.9$ years and $37.9 \pm 5.5$ years, respectively. None of the patients with ovarian endometrioma underwent hormonal treatment before surgery. Among the 47 patients with extragenital endometriosis, 21 patients did not undergo hormonal therapy, whereas 18 , 6 , and 2 patients were administered GnRH agonist, dienogest, and oral contraceptives, respectively, before surgery.

\section{General Features}

PAX8 was strongly positive in epithelial cells with the uniformly negative staining for stromal cells in ovarian endometrioma (Figure 1A). The staining of PAX8 was exclusively nuclear, with no expression in the stroma of extragenital endometriosis (Figure 1B and C). Moreover, PAX8 was negative for intestinal epithelia (Figure 1B and C). On the other hand, stromal cells were positive for CD10, whereas epithelial cells were negative for CD10 (Figure 1D). The expression of ER (Figure 1E) and PR (Figure 1F) was moderate to strong in the glands or the stroma.

\section{Ovarian Endometrioma}

For PAX8, the strong nuclear staining was seen in the epithelial cells of ovarian endometriosis ( 8 of $8,100 \%$ ), but not in the 


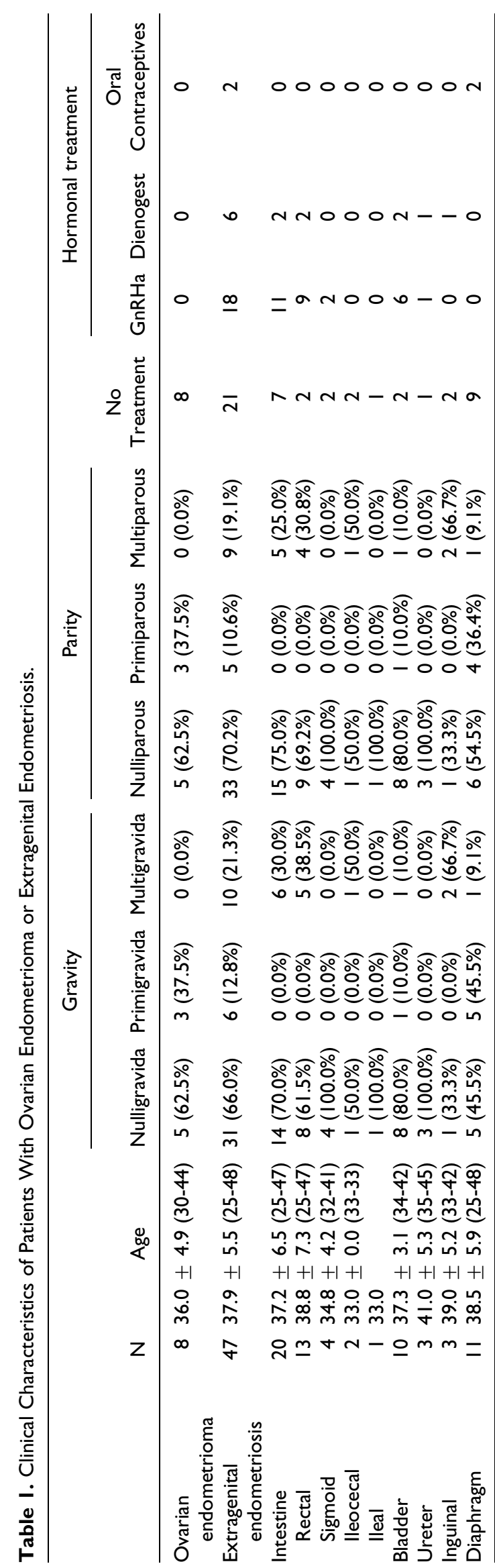

stromal cells ( 0 of $8,0 \%$; Table 2 ; Figure $2 \mathrm{~B})$. On the other hand, CD10 was positive in the stromal cells ( 8 of $8,100 \%)$, but not in the epithelial cells $(0$ of $8,0 \%)$ of ovarian endometriosis (Figure 2C). All ovarian endometrioses were immunoreactive for ER in the epithelial cells; of these, $5(62.5 \%)$ were immunoreactive in the stromal cells (Figure 2D). Progesterone receptor was positive in the epithelial and stromal cells of ovarian endometrioma ( 8 of $8,100 \%$; Figure 2E).

\section{Intestinal Endometriosis}

Twenty samples of intestinal endometriosis were examined in this study, which included 13 rectal endometrioses, 4 sigmoid endometrioses, 2 ileocecal endometrioses, and 1 ileal endometriosis. All of the $20(100.0 \%)$ intestinal endometrioses were immunoreactive in epithelia for PAX8, and none in the stroma $(0 / 20$; Figure $2 \mathrm{~F}$ and G; Supplemental Figure 1A and B). As for CD10, 19 of 20 (95.0\%) intestinal endometrioses were positive in the stroma and none in the epithelia (0/20; Figure 2H, Supplemental Figure 1C). The expression of ER (Figure 2I, Supplemental Figure 1D) and PR (Figure 2J, Supplemental Figure 1E) was frequently positive in the epithelial cells $(16 / 20,80.0 \%$ and $18 / 20$, $90.0 \%$, respectively) than in the stromal cells $(70.0 \%$ [14/ $20]$ and $70.0 \%$ [14/20], respectively). One case of intestinal endometriosis was positive for CD10 and PR and weakly positive for ER only in the glands but was strongly positive for PAX8 in the epithelial nucleus of the endometriosis (Figure 2K-O).

\section{Bladder and Ureteral Endometrioses}

Ten bladder and 3 ureteral endometrioses were examined. Eight of the $10(80.0 \%)$ bladder endometrioses and all $(100 \%)$ ureteral endometriosis were immunoreactive for PAX8 in the epithelial cells, but not in the stromal cells $(0 / 10,0.0 \%$ and $0 / 3,0.0 \%$, respectively; Supplemental Figure $2 \mathrm{~A}$ and $\mathrm{B}$ ). All bladder and ureteral endometriosis were immunoreactive for CD10 in the stromal cells $(10 / 10,100 \%$ and $3 / 3,100 \%$, respectively), but not in the epithelial cells $(0 / 10,0 \%$ and $0 / 3,0 \%$, respectively; Supplemental Figure 2C). Eight of the 10 bladder endometrioses were immunoreactive for ER in the epithelial cells ER, whereas 5 (50.0\%) of the 10 bladder endometrioses were immunoreactive in the stromal cells (Supplemental Figure 2D). One of the 3 ureteral endometrioses was positive in the epithelial cells $(1 / 3$, $33.3 \%)$, but none in the stromal cells $(0 / 3,0 \%)$. All of the 10 bladder endometrioses were positive for PR in the epithelial cells, and 2 of the 3 bladder endometrioses were positive for PR in the stromal cells (Supplemental Figure $2 \mathrm{E})$. Two $(66.6 \%)$ of the 3 ureteral endometrioses were immunoreactive for PR in the epithelial cells, and 1 $(33.3 \%)$ of the 3 ureteral endometrioses was positive for PR in the stromal cells. 

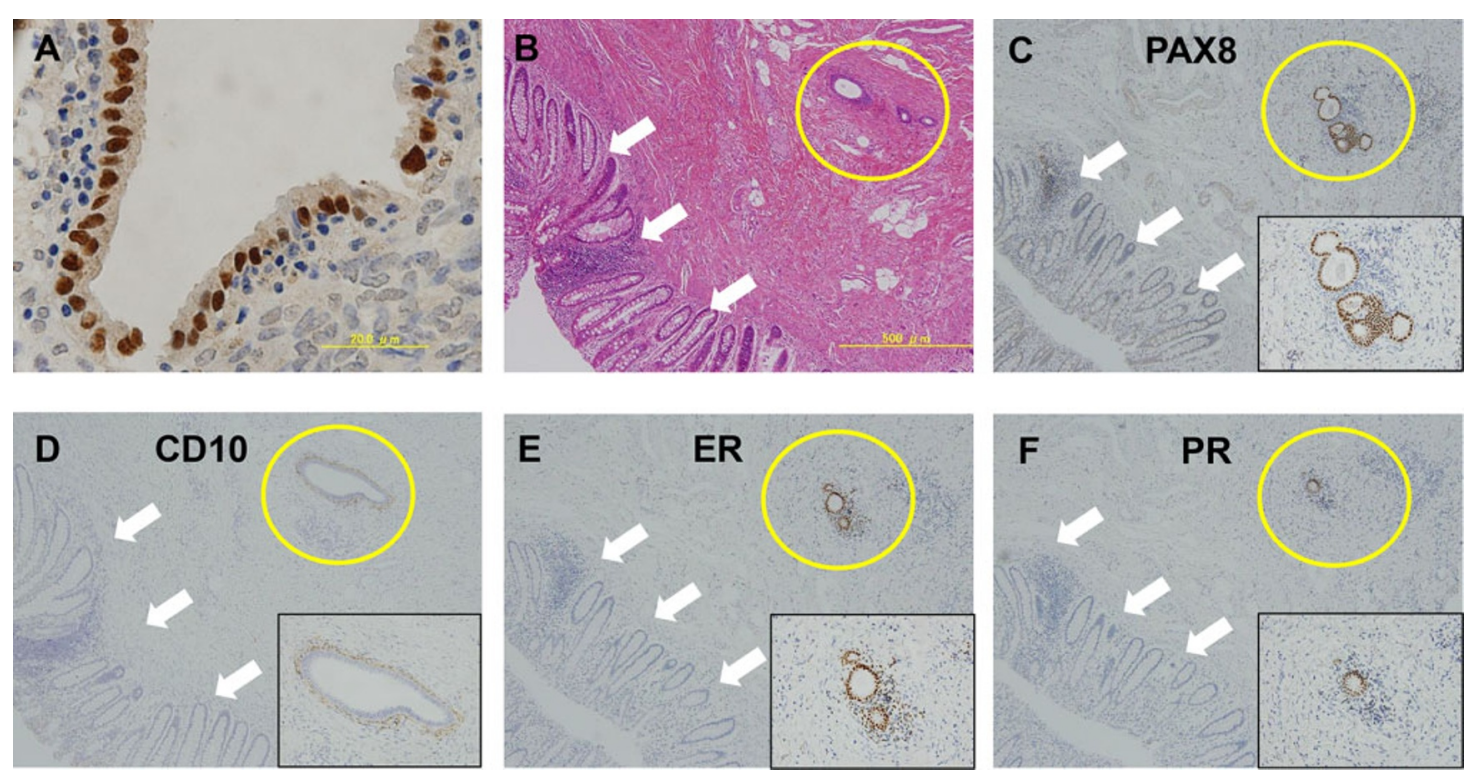

Figure I. Expression pattern of PAX8, CDI0, ER, and PR in ovarian endometrioma and extragenital endometriosis. (A) Ovarian endometrioma: PAX8 expression is limited to the nuclei of the glands. B-F: H\&E (B), PAX8 (C), CDI0 (D), ER (E), and PR (F) staining of rectal endometriosis. The insets show high power magnification images of endometriotic lesion. PAX8 is positive in the glands of endometriosis (yellow circle) but negative in the intestinal epithelia (white arrow). ER, estrogen receptor; H\&E, hematoxylin and eosin; PR, progesterone receptor.

\section{Inguinal Endometriosis}

PAX 8 was seen in the epithelial cells ( 3 of $3,100 \%)$, but not in the stromal cells $(0$ of $3,0 \%)$ of inguinal endometriosis (Supplemental Figure 3A, B). CD10 was positive in the stromal cells (3 of $3,100 \%)$, but not in the epithelial cells $(0$ of $3,0 \%)$ of inguinal endometriosis (Supplemental Figure 3C). All (100\%) inguinal endometrioses were immunoreactive for ER both in the epithelial and stromal cells (Supplemental Figure 3D). Progesterone receptor was positive in the epithelial and stromal cells of ovarian endometrioma (3 of 3, 100\%; Supplemental Figure 3E).

\section{Diaphragmatic Endometriosis}

Eleven diaphragmatic endometrioses were examined for immunostaining. Diaphragmatic endometriosis was immunoreactive for PAX8 in the epithelial cells $(11 / 11,100 \%)$, but not in the stromal cells $(0 / 11,0 \%$; Supplemental Figure 4B). The stroma of diaphragmatic endometriosis was positive for CD10 $(11 / 11,100 \%)$, and the epithelia was negative for CD10 (0/11; Supplemental Figure 4C). The expression of ER and PR was positive in the stromal cells $(11 / 11,100 \%$ and $11 / 11,100 \%$, respectively; Supplemental Figure 4D) and frequently positive in the epithelial cells at $90.9 \%(10 / 11)$ and $90.9 \%(10 / 11)$, respectively (Supplemental Figure 4E).

\section{The Effect of Hormonal Treatment on PAX8, CDIO, $E R$, or PR Expression}

The positive staining rates of PAX 8, CD10, ER, and PR were compared between the groups with and without hormonal therapy. Among the 47 patients, 26 patients underwent preoperative hormonal therapy, whereas 21 patients did not. As shown in Table 3, no significant difference was observed in the positive frequency of PAX8, CD10, and PR. On the other hand, the positive rate of ER expression was significantly reduced in the gland $(18 / 26,69.2 \%)$ and stroma (14/26, $53.8 \%$ ) by preoperative hormonal therapy.

\section{Discussion}

In this study, expression of PAX8, CD10, ER, and PR in extragenital endometriosis was examined comprehensively. Firstly, PAX8 was positive for endometriotic epithelial cells in $95.7 \%$ $(45 / 47)$ of extragenital endometriosis and in $100 \%(8 / 8)$ of ovarian endometriosis. Secondly, CD10 was positive for endometriotic stromal cells in 97.9\% (46/47) of extragenital endometriosis. Thirdly, the expression of PAX8, CD10, and PR was not affected by preoperative hormonal therapy, and the positive rate of ER staining was significantly reduced by preoperative hormonal therapy. Accordingly, PAX8 may be a useful sensitive marker for endometriotic epithelial cells, which is not affected by hormonal therapy. This is also the first report that comprehensively examined the immunostaining of extragenital endometriosis.

PAX8 was previously reported to be expressed in Mullerian epithelial cells (endometrium, endocervix, and fallopian tube). ${ }^{11,}{ }^{13}$ For the endometrium, PAX8 was positive in the epithelium in $100 \%$ of 35 cases. ${ }^{12,13}$ These reports showed that PAX8 is a sensitive marker in the endometrial epithelial cells. Additionally, it was reported that PAX8 was positive in $100 \%$ 


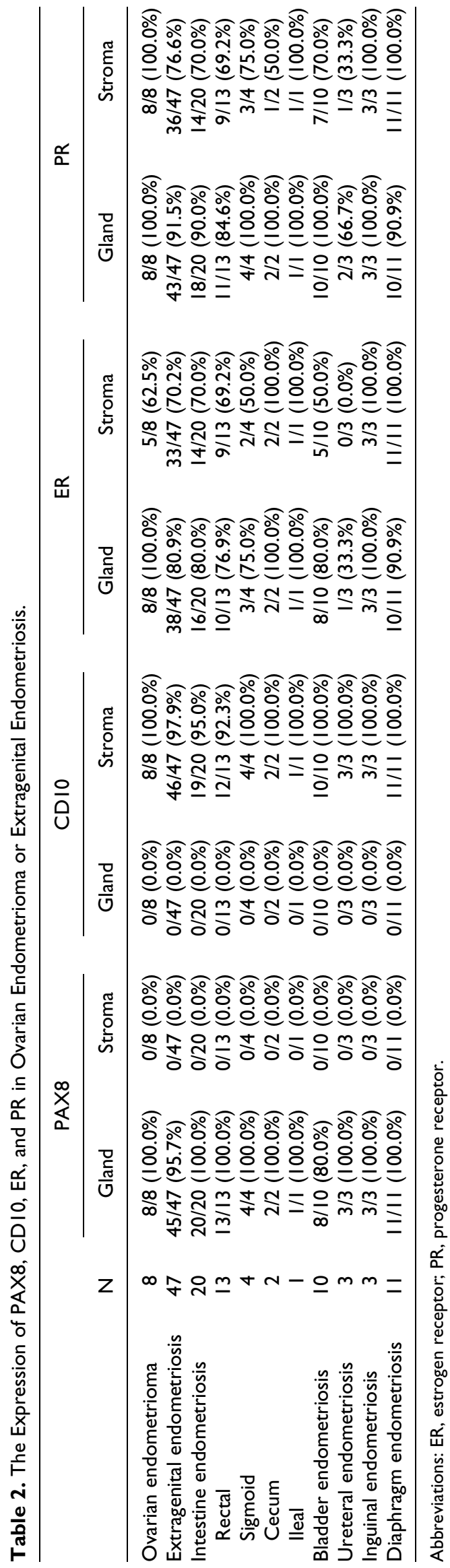

of 10 cases of endometriosis or adenomyosis, ${ }^{12,13}$ which is consistent with the results of our 8 ovarian endometrioma cases. Here, we demonstrated that PAX8 was positive in $95.7 \%$ of extragenital endometrioses, including rectosigmoid, bladder, ureteral, inguinal, and diaphragm endometrioses, which indicates that it is a useful diagnostic marker.

CD10 was positive in $100 \%$ (8/8) of ovarian endometrioma and in $97.9 \%$ (46/47) of extragenital endometriosis, which indicates that CD10 is also useful as an endometriotic stromal marker, consistent with previous reports. ${ }^{2,3}$ The thoracic endometriotic stromal cells were also reported to be positive for CD10 in $88.1 \%$ of the samples. ${ }^{15}$ Moreover, the ureteral endometriosis was positive for CD10 in $100 \%$ of 6 cases. ${ }^{16}$ In our case series, there was one case in which CD10 staining was negative, PR was negative, and ER was slightly positive. However, PAX8 was strongly positive for the glands. Considering that the glands can sometimes be observed in the fibromuscular tissue without any surrounding stroma, ${ }^{4,17}$ it is useful to confirm the diagnosis of endometriosis by performing PAX8 staining.

In the previous report, rectosigmoid, umbilical, and ureteral endometrioses were reported to show nuclear staining for ER and PR in the glands or stroma. ${ }^{16,18,19}$ Estrogen receptor or PR was positive in the glands of thoracic endometriosis in $85.7 \%$ or $85.7 \%$ of cases, respectively. ${ }^{15}$ In this study, ER or PR was positive in the glands at $80.9 \%$ (38/47) or $91.5 \%$ (43/ 47) of extragenital endometrioses, respectively. However, PAX8 was a marker of endometriotic epithelial cells, which was comparable or even more sensitive than these hormone receptors. In particular, lesions of thoracic endometriosis are small in size and often difficult to diagnose. ${ }^{15}$ Furthermore, there has been also a report that the presence of glands was associated with postoperative recurrence, ${ }^{20}$ and it is considered that the identification of endometriotic epithelium is clinically important for postoperative management of thoracic endometriosis.

We also confirmed that PAX8 functions as an endometriotic epithelial marker in patients under hormonal therapy, such as GnRH agonist and dienogest. On the other hand, the positive rate of ER staining was significantly reduced under hormonal therapy. Consistent with our result, previous reports demonstrated that progestin and GnRH agonist reduced the expression of ER in the epithelium and stroma of endometriosis and endometrium. ${ }^{21,22}$ These reports also indicated that the expression of PR was also reduced by these hormonal therapies, although it was not affected in our study. When surgery is required for the extragenital endometriosis, preoperative hormonal therapy using GnRH agonist or dienogest is often performed to reduce the size of lesion or control the disease condition and symptoms. Therefore, it is important as an endometriosis marker that the expression of PAX8 and CD10 was not affected by these hormonal therapies.

PAX8 is a lineage marker for Mullerian lineage epithelial cells, including normal/nonneoplastic Mullerian epithelial cells and Mullerian primary and metastatic tumor. ${ }^{11-14}$ Our examination demonstrated that extragenital endometriosis also 


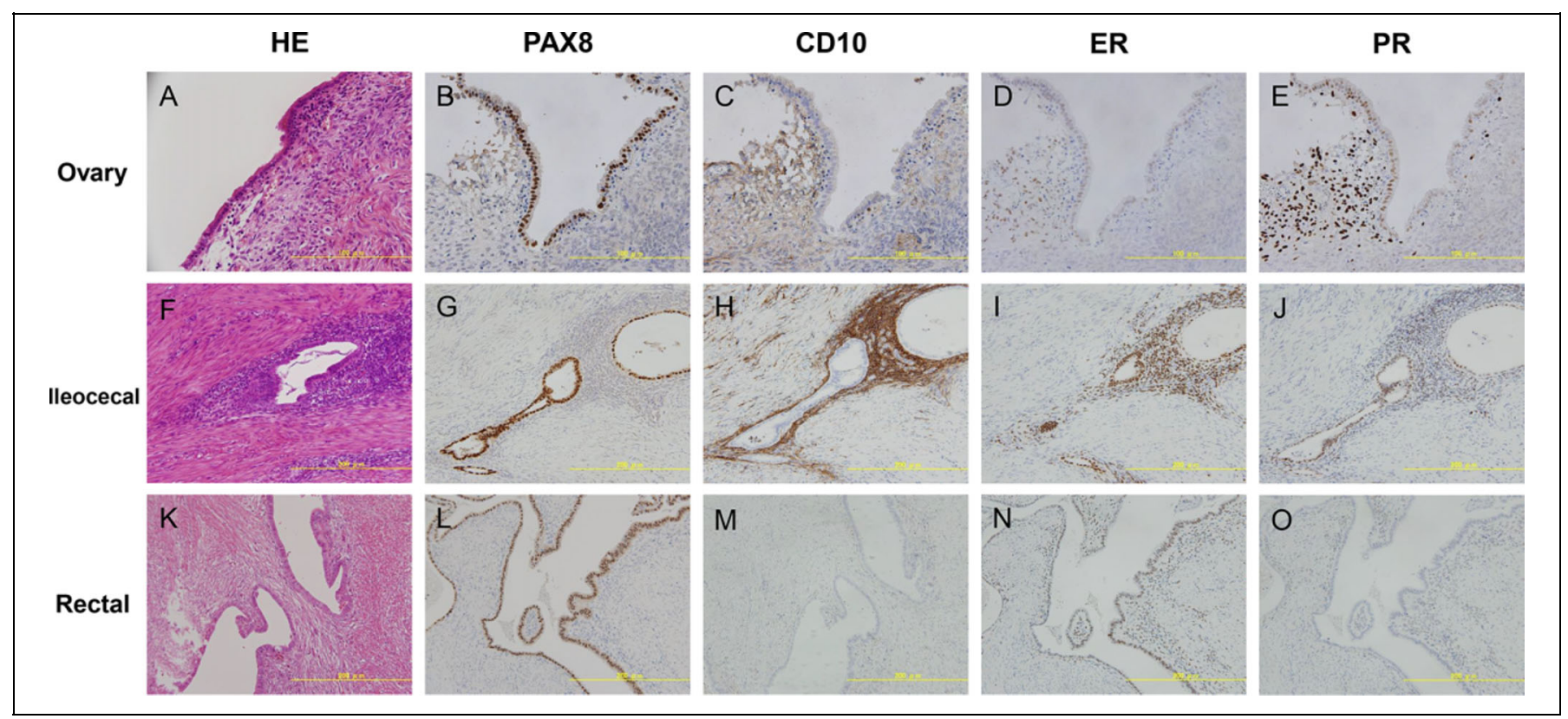

Figure 2. $P A X 8, C D I 0, E R$, and $P R$ expression in ovarian endometrioma and extragenital endometriosis. $H \& E(A), P A X 8$ (B), CDI0 (C), ER (D), and PR(E) staining in ovarian endometrioma. PAX8 is positive in the nuclei in the glands. CDIO is positive in the stromal cells. ER and $P R$ are moderately to strongly positive in the glands and stroma. H\&E (F), PAX8 (G), CDI0 (H), ER (I), and PR (J) staining in ileocecal endometrioma. PAX8 is strongly positive in the nuclei of the glands. CDIO is strongly positive in the stroma. ER and PR are positive in the nuclei of the glands and stroma. H\&E (K), PAX8 (L), CDIO (M), ER (N), and PR (O) staining in rectal endometriosis. (K) The glands are observed in the fibromuscular tissue without any surrounding stroma. (L) PAX8 is strongly positive in the nuclei of glands. CDIO (M) and PR(O) are negative. $(\mathrm{N})$ Estrogen receptor is moderately positive in the nuclei of glands and fibromuscular tissue. ER indicates estrogen receptor; $\mathrm{H} \& \mathrm{E}$, hematoxylin and eosin; PR, progesterone receptor.

Table 3. The Comparison of PAX8, CDI0, ER and PR Expression in Extragenital Endometriosis With or Without Hormonal Treatment.

\begin{tabular}{|c|c|c|c|c|c|c|}
\hline & Without Hormonal Treatment & With Hormone Treatment & $P$ Value & GnRH Agonist & Dienogest & Oral Contraceptives \\
\hline \multicolumn{7}{|l|}{ PAX8 } \\
\hline gland & $2 \mathrm{I} / 2 \mathrm{I}(100.0 \%)$ & $24 / 26(92.3 \%)$ & NS & 16/18 (88.9\%) & $6 / 6(100.0 \%)$ & $2 / 2(100.0 \%)$ \\
\hline \multicolumn{7}{|l|}{ CDIO } \\
\hline gland & $0 / 21(0.0 \%)$ & $0 / 26(0.0 \%)$ & NS & $0 / 18(0.0 \%)$ & $0 / 6(0.0 \%)$ & $0 / 2(0.0 \%)$ \\
\hline Stroma & $20 / 21(95.2 \%)$ & $26 / 26(100.0 \%)$ & NS & $18 / 18(100.0 \%)$ & $6 / 6(100.0 \%)$ & $2 / 2(100.0 \%)$ \\
\hline Stroma & $19 / 21(90.5 \%)$ & $14 / 26(53.8 \%)$ & $P<.01$ & $10 / 18(55.6 \%)$ & $2 / 6(33.3 \%)$ & $2 / 2(100.0 \%)$ \\
\hline \multicolumn{7}{|l|}{ PR } \\
\hline Gland & $|7 / 2|(8 \mid .0 \%)$ & $24 / 26(92.3 \%)$ & NS & $18 / 18(100.0 \%)$ & $4 / 6(66.7 \%)$ & $2 / 2(100.0 \%)$ \\
\hline Stroma & $|7 / 2|(8 \mid .0 \%)$ & $18 / 26(69.2 \%)$ & NS & $12 / 18(66.7 \%)$ & $4 / 6(66.7 \%)$ & $2 / 2(100.0 \%)$ \\
\hline
\end{tabular}

Abbreviations: ER, estrogen receptor; NS, not significant; PR, progesterone receptor.

maintained a lineage marker. PAX 8 is embryologically an essential molecule for the development of Mullerian organs. ${ }^{10}$ PAX8 is also an essential transcriptional factor for the thyroid gland and urinary tract system. ${ }^{8,9}$ In the PAX8 ablation mice, the urinary tract system is not affected and the endometrium is deficient and becomes infertile. ${ }^{10}$ Furthermore, there has been a case report of a patient with Mullerian agenesis attributed to the heterozygous deficiency of PAX $8 .{ }^{23}$ Therefore, PAX8 plays an important role in the development and function of Mullerian organs, such as the endometrium, not only in mice but also in humans. From this aspect, it is noteworthy that not only ovarian endometriosis but also extragenital endometriosis maintains the expression of this lineage marker, although they occur at various sites, and their cause and mechanism of development might be different.

As a limitation of this study, other extragenital endometrioses, such as umbilical and abdominal wall endometrioses, were not examined. However, to our knowledge, it is the first study to comprehensively examine cases with extragenital endometriosis in various sites. 
In conclusion, this study shows that PAX8 is a highly sensitive epithelial marker for extragenital endometriosis. This specific expression was maintained under hormonal therapy. PAX8 nuclear expression can be useful in detecting extragenital endometriosis in clinical practice.

\section{Authors' Note}

Tomoko Arakawa, MD, and Shinya Fukuda, MD, $\mathrm{PhD}$, are joint first authors.

\section{Acknowledgments}

We would like to thank Editage (www.editage.jp) for the English language review.

\section{Declaration of Conflicting Interests}

The author(s) declared no potential conflicts of interest with respect to the research, authorship, and/or publication of this article.

\section{Funding}

The author(s) disclosed receipt of the following financial support for the research, authorship, and/or publication of this article: This work was supported by grants from the Ministry of Health, Labour and Welfare, the Ministry of Education, Culture, Sports, Science and Technology and the Japan Agency for Medical Research and Development.

\section{ORCID iD}

Tetsuya Hirata, MD, PhD (D) https://orcid.org/0000-0002-9975-6208

\section{Supplemental Material}

Supplemental material for this article is available online.

\section{References}

1. Zondervan KT, Becker CM, Koga K, Missmer SA, Taylor RN, Vigano P. Endometriosis. Nat Rev Dis Primers. 2018;4(1):9.

2. Sumathi VP, McCluggage WG. CD10 is useful in demonstrating endometrial stroma at ectopic sites and in confirming a diagnosis of endometriosis. J Clin Pathol. 2002;55(5):391-392.

3. Potlog-Nahari C, Feldman AL, Stratton P, et al. CD10 immunohistochemical staining enhances the histological detection of endometriosis. Fertil Steril. 2004;82(1):86-92.

4. Vigano P, Candiani M, Monno A, Giacomini E, Vercellini P, Somigliana E. Time to redefine endometriosis including its profibrotic nature. Hum Reprod. 2018;33(3):347-352.

5. Noe M, Ayhan A, Wang TL, Shih IM. Independent development of endometrial epithelium and stroma within the same endometriosis. J Pathol. 2018;245(3):265-269.

6. Mansouri A, Hallonet M, Gruss P. Pax genes and their roles in cell differentiation and development. Curr Opin Cell Biol. 1996;8(6): 851-857.

7. Dahl E, Koseki H, Balling R. Pax genes and organogenesis. Bioessays. 1997;19(9):755-765.

8. Plachov D, Chowdhury K, Walther C, Simon D, Guenet JL, Gruss P. Pax8, a murine paired box gene expressed in the developing excretory system and thyroid gland. Development. 1990;110(2):643-651.
9. Poleev A, Fickenscher H, Mundlos S, et al. PAX8, a human paired box gene: isolation and expression in developing thyroid, kidney and Wilms' tumors. Development. 1992;116(3):611-623.

10. Mittag J, Winterhager E, Bauer K, Grummer R. Congenital hypothyroid female pax8-deficient mice are infertile despite thyroid hormone replacement therapy. Endocrinology. 2007;148(2): 719-725.

11. Laury AR, Perets R, Piao H, et al. A comprehensive analysis of PAX8 expression in human epithelial tumors. Am J Surg Pathol. 2011;35(6):816-826.

12. Ozcan A, Liles N, Coffey D, Shen SS, Truong LD. PAX2 and PAX8 expression in primary and metastatic Mullerian epithelial tumors: a comprehensive comparison. Am J Surg Pathol. 2011; 35(12): 1837-1847.

13. Ozcan A, Shen SS, Hamilton C, et al. PAX 8 expression in nonneoplastic tissues, primary tumors, and metastatic tumors: a comprehensive immunohistochemical study. Mod Pathol. 2011;24(6): 751-764.

14. Ordonez NG. Value of PAX 8 immunostaining in tumor diagnosis: a review and update. Adv Anat Pathol. 2012;19(3):140-151.

15. Haga T, Kumasaka T, Kurihara M, Kataoka H, Miura M. Immunohistochemical analysis of thoracic endometriosis. Pathol Int. 2013;63(9):429-434.

16. Al-Khawaja M, Tan PH, MacLennan GT, Lopez-Beltran A, Montironi R, Cheng L. Ureteral endometriosis: clinicopathological and immunohistochemical study of 7 cases. Hum Pathol. 2008; 39(6):954-959.

17. Donnez J, Nisolle M, Casanas-Roux F, Brion P, Da Costa Ferreira N. Stereometric evaluation of peritoneal endometriosis and endometriotic nodules of the rectovaginal septum. Hum Reprod. 1996; 11(1):224-228.

18. Zanatta A, Pereira RM, Rocha AM, et al. The relationship among HOXA10, estrogen receptor alpha, progesterone receptor, and progesterone receptor B proteins in rectosigmoid endometriosis: a tissue microarray study. Reprod Sci. 2015;22(1):31-37.

19. Colon-Caraballo M, Garcia M, Mendoza A, Flores I. Human endometriosis tissue microarray reveals site-specific expression of estrogen receptors, progesterone receptor, and Ki67. Appl Immunohistochem Mol Morphol. Epub ahead of print 7 April 2018.

20. Haga T, Kurihara M, Kataoka H, Ebana H. Clinical-pathological findings of catamenial pneumothorax: comparison between recurrent cases and non-recurrent cases. Ann Thorac Cardiovasc Surg. 2014;20(3):202-206.

21. Gomes MK, Rosa-e-Silva JC, Garcia SB, et al. Effects of the levonorgestrel-releasing intrauterine system on cell proliferation, Fas expression and steroid receptors in endometriosis lesions and normal endometrium. Hum Reprod. 2009;24(11):2736-2745.

22. Kitano T, Matsumoto T, Takeuchi H, et al. Expression of estrogen and progesterone receptors in smooth muscle metaplasia of rectovaginal endometriosis. Int J Gynecol Pathol. 2007;26(2): 124-129.

23. Ma D, Marion R, Punjabi NP, et al. A de novo $10.79 \mathrm{Mb}$ interstitial deletion at 2q13q14.2 involving PAX8 causing hypothyroidism and Mullerian agenesis: a novel case report and literature review. Mol Cytogenet. 2014;7(1):85. 